\title{
Estudio experimental para la valoración de la cicatrización ósea con Ácido Zoledrónico en ratas Wistar
}

\author{
Moñivas Palomero C. ${ }^{1}$, García López P. ${ }^{2 a}$, Arias Sanz P.3a , Izquierdo Hernández A. ${ }^{\text {aa }}$, López Carrizosa C.5a, \\ Guijarro Sánchez D. ${ }^{6}$
}

Sanid. mil. 2015; 71 (4): 232-238; ISSN: 1887-8571

\begin{abstract}
RESUMEN
Antecedentes y objetivos: El aumento del consumo de bifosfonatos en la sociedad actual, puede incrementar el riesgo de osteonecrosis mandibular. Se realizó este estudio para valorar si tras la extracción dentaria, después de la administración subcutánea de ácido zoledrónico 7,5 $\mu \mathrm{g} / \mathrm{Kg}$ o $35 \mu \mathrm{g} / \mathrm{Kg}$ (Zometa $\left.{ }^{\circledR}\right)$ en ratas Wistar, aparecen signos clínicos, radiográficos e histopatológicos de osteonecrosis y/o inflamación. Lugar de realización: Centro Experimentación Animal del Hospital de Defensa. Material y Métodos: Estudio experimental, in vitro, randomizado, intervencionista. Se utilizaron un total de 30 ratas Wistar (adultas, sanas), repartiéndolas en tres grupos de 10 animales, según sexo, grupo y fármaco: $\mathrm{G}_{0}$ : Sin tratamiento con ácido zoledrónico. $\mathrm{G}_{1}$ : Con tratamiento de ácido zoledrónico 7,5 $\mathrm{\mu g} / \mathrm{Kg}$ subcutáneo una dosis en los días 1,15 y 30 . $\mathrm{G}_{2}$ : Con tratamiento de ácido zoledrónico $35 \mu \mathrm{g} / \mathrm{Kg}$ subcutáneo una dosis en los días 1, 15 y 30. En todos los grupos se realizó exodoncia del primer molar inferior derecho el día 30, sacrificando los animales a las cuatro semanas postextracción, observando clínica, histológica y radiográficamente la aparición de osteonecrosis e inflamación. Resultados: Clínicamente se observaron en un $26,6 \%$ falta de epitelización compatible con signos precoces de osteonecrosis mandibular, según criterios de la American Association of Oral Maxillofacial Súrgeons (AAOMS). Esta es dosis dependiente en 3 animales de $\mathrm{G}_{1}(10 \%)$ y 5 animales de $\mathrm{G}_{2}(16,6 \%)$. Los resultados presentaron significación estadística p $<0,011$. Ausencia histológica y radiológica de osteonecrosis $\mathrm{p}<0,001$ e inflamación $\mathrm{p}<0,001$ en todos los grupos. Conclusiones: La administración subcutánea de 7,5 $\mu \mathrm{g} / \mathrm{Kg}$ o $35 \mu \mathrm{g} / \mathrm{Kg}$ de ácido zoledrónico durante cuatro semanas, tras la realización de una extracción dentaria, no da lugar a signos histopatológicos de osteonecrosis e inflamación $(\mathrm{p}<0,001)$ pero si a alteraciones clínicas dosis dependientes $(\mathrm{p}<0,011)$ compatibles con estadios iniciales de osteonecrosis mandibular según criterios de la AAOMS.
\end{abstract}

PALABRAS CLAVE: Bifosfonatos, ácido zoledrónico, osteonecrosis, extracción dental, ratas.

\section{Experimental studies for valuing bone healing on Wistar rats with Zoledronic Acid}

SUMMARY: Records and objectives: The increase of biphosphonates consumption on current society may increase the risk of mandibular osteonecrosis. This study was developed in order to value if, after dental extraction with a subcutaneous administration of zoledronic acid 7,5 $\mu \mathrm{g} / \mathrm{Kg}$ or $35 \mu \mathrm{g} / \mathrm{Kg}\left(\right.$ Zometa $\left.{ }^{\circledR}\right)$ on Wistar rats, any clinic, radiographic or histopathological evidence of osteonecrosis or inflammation appear. Place of execution: Animal Experimentation Centre of the Hospital of Defence. Materials and methods: Experimental study, in vitro, randomized interventionist. A total amount of 30 Wistar rats were used (adults and healthy), divided into 3 groups of 10 animals according to sex, group and medicine. $\mathrm{G}_{0}$ : no Zoledronic Acid treatment. $\mathrm{G}_{1}$ : Zoledronic Acid treatment 7,5 $\mu \mathrm{g} / \mathrm{Kg}$ subcutaneous, one dose on days 1 , 15 and $30 . \mathrm{G}_{2}$ : Zoledronic Acid treatment $35 \mu \mathrm{g} / \mathrm{Kg}$ subcutaneous, one dose on days 1, 15 and 30 . On all the groups an extraction of the lower right first molar was done on day 30, killing the animals four weeks post-extraction, observing clinically, histologically and radiographically the appearance of osteonecrosis and inflammation. Results: Clinically, a 26,6\% showed a lack of epithelization compatible with early signs of mandibular osteonecrosis, according to the American Association of Oral Maxillofacial Surgeos (AAOMS) criteria. This is a dependent dose on 3 animals from G1 (10\%) and 5 animals from G2 (16,6\%). These results presented statistic signification p $<0,011$. Histological and radiological absence of osteonecrosis $\mathrm{p}<0,001$ and inflammation $\mathrm{p}<0,001$ in all the groups. Conclusion: Subcutaneous administration $7,5 \mu \mathrm{g} / \mathrm{Kg}$ or $35 \mu \mathrm{g} /$ $\mathrm{Kg}$ of Zoledronic Acid during four weeks, after the dental extraction, does not lead to histopathological signs of osteonecrosis and inflammation $(\mathrm{p}<0,001)$ but leads to clinical alterations dose-dependent $(\mathrm{p}<0,011)$ compatible with early stages of mandibular osteonecrosis according to AAOMS criteria.

KEYWORDS: Bisphosphonate, zoledronic acid, osteonecrosis, dental extraction, rats.

${ }^{1}$ Licenciada en Odontología. Universidad Europea de Madrid. Facultad de Ciencias Biomédicas. Dpto de Odontología de Adultos.

Tte. Veterinario. Servicio Cirugía Experimental.

3 Tcol. Veterinario. Servicio Cirugía Experimental.

${ }^{4}$ Facultativo especialista de Área. Servicio de Anatomía Patológica.

5 Facultativo especialista de Área. Servicio de Oncología Radioterápica.

6 Técnico Especialista en Higiene Bucodental. Servicio de Odontología de la Unidad de Apoyo. Centro de Salud Ramón y Cajal.

Hospital Central de la Defensa Gómez Ulla. Madrid.

Dirección para correspondencia: Carolina Moñivas Palomero. Facultad de Ciencias Biomédicas. Universidad Europea de Madrid. C/Tajo s/n. C.P 28670 Villaviciosa de Odón (Madrid). E-mail: carolina.monivas@uem.es

Recibido: 6 de marzo de 2015

Aceptado: 10 de junio de 2015

\section{INTRODUCCIÓN}

Los bifosfonatos (BF) son medicamentos sintéticos análogos del pirofosfato inorgánico, con elevada afinidad por el calcio, cuya función es inhibir la actividad de los osteoclastos, tener efecto antiangiogénico y antitumoral ${ }^{1-4}$.

Se utilizan principalmente para la prevención y tratamiento de enfermedades que cursan con reabsorción ósea, como la osteoporosis, osteogénesis imperfecta, enfermedad de Paget, metástasis ósea, mieloma múltiple e hipercalcemia maligna inducida por tumores entre otras ${ }^{4-7}$. 
Estudio experimental para la valoración de la cicatrización ósea con Ácido Zoledrónico en ratas Wistar

Tabla 1. Clasificación de los Bifosfonatos ${ }^{7-11}$.

\begin{tabular}{|c|c|c|c|c|c|c|}
\hline Potencia & $\begin{array}{l}\text { Principio } \\
\text { activo }\end{array}$ & Nombre comercial & $\begin{array}{l}\text { Contiene } \\
\text { nitrógeno }\end{array}$ & $\begin{array}{l}\text { Genera- } \\
\text { ción }\end{array}$ & Vía & Utilización \\
\hline$>10.000$ & Zoledronato & $\begin{array}{l}\text { Zometa } \\
\text { Aplasta }\end{array}$ & $\mathrm{Si}$ & $3^{\circ}$ & I.V & $\begin{array}{l}\text { Osteoporosis. Enfermedad de Paget. Cáncer de } \\
\text { mama y de próstata. }\end{array}$ \\
\hline $\begin{array}{c}>10000-< \\
1000\end{array}$ & Risendronato & $\begin{array}{l}\text { Actonel } \\
\text { Acrel } \\
\text { Losentra }\end{array}$ & $\mathrm{Si}$ & $3^{\circ}$ & Oral & $\begin{array}{l}\text { Osteoporosis. Enfermedad de Paget. Cáncer de } \\
\text { mama. Cáncer de próstata. Mieloma Múltiple. }\end{array}$ \\
\hline $\begin{array}{c}>10000-< \\
1000\end{array}$ & Ibandronato & $\begin{array}{l}\text { Bondronat } \\
\text { Boniva } \\
\text { Bonviva }\end{array}$ & $\mathrm{Si}$ & $3^{\circ}$ & $\begin{array}{l}\text { Oral } \\
\text { I.V }\end{array}$ & $\begin{array}{l}\text { Osteoporosis. Cáncer de mama. } \\
\text { Hipercalcemia inducida por tumor con o sin } \\
\text { metástasis. }\end{array}$ \\
\hline$>1000-<100$ & Alendronato & $\begin{array}{l}\text { Fosamax } \\
\text { Fosavance } \\
\text { Adronat }\end{array}$ & $\mathrm{Si}$ & $2^{\circ}$ & Oral & $\begin{array}{l}\text { Osteoporosis postmeneopáusica. } \\
\text { Tratamiento y profilaxis osteoporosis inducida por } \\
\text { corticoides. } \\
\text { Enfermedad de Paget. }\end{array}$ \\
\hline 100 & Pamidronato & $\begin{array}{l}\text { Aredia } \\
\text { Linoten } \\
\text { Pamifos }\end{array}$ & $\mathrm{Si}$ & $2^{\circ}$ & I.V & $\begin{array}{l}\text { Hipercalcemia inducida por tumor. Enfermedad } \\
\text { de Paget (refractaria a otros ttos). Metástasis óseas } \\
\text { osteolíticas en cáncer de mama. Mieloma Múltiple. }\end{array}$ \\
\hline 10 & Tiludronato & Skelid & No & $1^{\mathrm{o}}$ & Oral & Enfermedad de Paget. \\
\hline 10 & Clodronato & $\begin{array}{l}\text { Bonefos } \\
\text { Loron } \\
\text { Mebonat } \\
\text { Hemocalcin }\end{array}$ & No & $1^{\mathrm{o}}$ & $\begin{array}{l}\text { Oral } \\
\text { I.V }\end{array}$ & $\begin{array}{l}\text { Cáncer de mama. Hipercalcemia } 2^{\mathrm{a}} \text { a tumores } \\
\text { óseos primarios o metastáticos. Metástasis óseas } \\
\text { osteolíticas de tumores sólidos o de neoplasias } \\
\text { hematológicas. }\end{array}$ \\
\hline 1 & Etidronato & $\begin{array}{l}\text { Didronel } \\
\text { Disfosfen } \\
\text { Osteum }\end{array}$ & No & $1^{\mathrm{o}}$ & Oral & $\begin{array}{l}\text { Enfermedad de Paget. Osteoporosis } \\
\text { postmeneopáusica. } \\
\text { Osificaciones heterotópicas }\end{array}$ \\
\hline
\end{tabular}

Los BF pueden clasificarse según su composición química, generación y vía de administración, tal y como se observa en la Tabla $1^{7-11}$.

\section{Composición Química}

Los que contienen nitrógeno y los que no tienen nitrógeno. Los BF que contienen nitrógeno, tienen mayor capacidad inhibitoria 8,11 , acumulándose en el hueso, con fuerte actividad antiosteoclásti$\mathrm{ca}^{12,13}$, inhibiendo las metástasis óseas y reduciendo la carga tumoral de los tumores óseos ${ }^{5,6,11}$.

\section{Generación}

Primera Generación, lo forman algunos bifosfonatos no nitrogenados, debido a su similitud estructural cercana al pirofosfato inorgánico.

Segunda Generación, inhiben la reabsorción ósea a unas dosis que no afecta la mineralización, siendo más potentes que los de la primera generación.

Tercera Generación, son bifosfonatos nitrogenados mucho más potentes $^{10,12,13}$

\section{Vía de administración}

Vía Intravenosa: Si se administra por vía intravenosa, aproximadamente el 50-75\% de la dosis inyectada se une fuertemente al hueso, donde se une al osteoclasto y el resto es excretado por el riñón ${ }^{14}$. Esta vía de administración se utiliza principalmente para pacientes con cáncer y está asociado con el mayor riesgo de desarrollar osteonecrosis mandibular o maxilar ${ }^{8,15}$.
Vía Oral: La administración por vía oral, sólo una pequeña proporción, aproximadamente $2 \%$, se absorbe en relación con los alimentos $^{16}$. Su potencia es menor y se utilizan principalmente para el tratamiento de la osteoporosis ${ }^{12,17}$.

La máxima acumulación de los BF se produce en la matriz ósea (intravenosa $50 \%$ y $2 \%$ oral) $)^{15,16}$, disminuyendo la actividad osteoclástica ${ }^{1}$.

La vida media de los bifosfonatos en circulación es corta, desde 30 minutos hasta 2 horas $^{13}$, fijándose en el tejido óseo durante muchos años ${ }^{7,9,12}$, y los que no han sido absorbidos, se eliminan rápidamente por el riñón ${ }^{7}$. Para el alendronato sugieren una vida media de más de 10 años después de una única dosis intravenosa ${ }^{7,9,12}$.

La relación existente entre bifosfonatos y osteonecrosis de los maxilares (ONMB) empezó a describirse a partir del año 2003, donde diferentes artículos sugerían la relación existente entre la toma de bifosfonatos y ostenocrosis, en pacientes que tenían cáncer $^{11,12,18}$. Desde entonces han surgido numerosos artículos que describen como los bifosfonatos, sobre todo por vía intravenosa, pueden desarrollar necrosis ósea avascular, actuando sobre metabolismo del calcio y fosfato, inhibiendo la formación de la angiogénesis ${ }^{2,3}$, disminuyendo la proliferación celular e induciendo la apoptosis ${ }^{3}$.

Actualmente el diagnóstico suele estar basado solo en la evaluación clínica, pero en estadios más avanzados se pueden observar hallazgos radiográficos e histológicos de la ONMB.

Los requisitos clínicos para el diagnóstico de ONMB son: Hallarse bajo tratamiento previo con bifosfonatos, existir hueso expuesto no cicatrizado en la región maxilofacial que persista más allá de ocho semanas, y no tener historia de radioterapia en los maxilares ${ }^{19}$.

Radiológicamente para determinar la ONMB son: la esclerosis ósea, la reacción perióstica y secuestro óseo ${ }^{20}$. 
Histológicamente, se pueden apreciar colonias bacterianas, hueso necrótico acelular, aumento del número de osteoclastos, resorción ósea prominente e infiltración inflamatoria masiva ${ }^{21}$.

La ONMB tiene mayor predilección en los maxilares que en otros huesos del organismo porque están expuestos a procesos infecciosos relacionados con los dientes y de los tejidos blandos, tienen una vascularización de tipo terminal y sufren microtraumatismos durante la masticación ${ }^{22,23}$. La localización más frecuente es la mandíbula (65-68\%), seguido por el maxilar superior $(26-27 \%)^{8,24}$, que es un hueso con mayor irrigación ${ }^{11,12,22,25,26}$. También se describen casos en ambos maxilares a la vez, pero es menos frecuente $(4,2-$ $9 \%)^{8,24}$

Esta lesión puede manifestarse de forma espontánea, sin embargo, el mayor riesgo de aparición de la ONMB se produce tras un trauma dentoalveolar. La exodoncia es el proceso quirúrgico odontológico más habitual. La exposición ósea que conlleva, posibilita la infección, produciendo dolor, inflamación, supuración y finalmente necrosis ósea ${ }^{8,12,17,24,25,27}$.

La etiopatogenia de la ONMB se desconoce, ya que es de origen multifactorial ${ }^{28}$. Sin embargo, se ha observado que el riesgo de desarrollar ONMB puede aumentar si el paciente presenta: enfermedades dentales inflamatorias ${ }^{27}$, cirugía oral ${ }^{24}$, tipo de $\mathrm{BF}^{22}$, duración del $\mathrm{BF}^{16,19,25,27}$, vía de administración ${ }^{7,8,15}$, enfermedades (mieloma múltiple, cáncer de mama y próstata) ${ }^{12,22,24,25}$, $\operatorname{sexo}^{15,19,22,25}$, edad ${ }^{15,22,25}$ y factores sistémicos $7,11,15$.

Actualmente, no existe un tratamiento efectivo y definitivo para los casos de $\mathrm{ONMB}^{25}$. Las alternativas terapéuticas dependen hasta el momento, de cada paciente, de su progresión y de la decisión del personal médico-odontológico responsable. En pacientes donde el tratamiento con antibioterapia no ha funcionado, se planifica el tratamiento quirúrgico, que puede incluir: desbridamiento, secuestrectomía, maxilectomía y mandibulectomía parcial o total ${ }^{8,25,29,30}$.

La confirmación clínica de la ONMB ha sido clasificada en cuatro grupos, según la American Association of Oral Maxillofacial Súrgeons (AAOMS) ${ }^{31}$ :

- Estadio 0: Sin evidencias de hueso expuesto en pacientes con tratamiento de BF orales o intravenosos. No requieren ningún tratamiento, pero deben ser informados respecto al riesgo que poseen de desarrollar una ONMB.

- Estadio 1: Presencia de hueso expuesto sano o necrótico en pacientes asintomáticos. No hay evidencia de infección o de inflamación significativa del tejido blando de los maxilares. Tratamiento con colutorios o geles de clorhexidina.

- Estadio 2: Presencia de hueso expuesto o necrótico en pacientes con dolor, con signos evidentes de infección secundaria e inflamación del tejido blando subyacente. Tratamiento clorhexidina, antiinflamatorios y antibióticos orales.

- Estadio 3: Presencia de hueso expuesto o necrótico en pacientes con dolor, inflamación, infección y uno o más de los siguientes signos: fractura patológica, fístula extra-oral $\mathrm{u}$ osteolisis que se extiende al borde inferior. Tratamiento clorhexidina, antiinflamatorios y antibióticos intravenosos.

El creciente consumo de BF, y la dificultad de tratamiento de los casos de osteonecrosis secundaria ${ }^{8,25,29,30,32}$, hacen razonable estudiar en modelos de investigación animal la aparición de ONMB. Comprender el mecanismo de producción y los factores coadyuvantes o facilitadores ayudaría a una mejor prevención, un pronto diagnóstico y un exitoso tratamiento.
Por ello, en este estudio experimental se valoró clínica, histológica y radiográficamente la aparición de osteonecrosis mandibular. El ensayo se realiza en 30 ratas sanas, 10 de las cuales recibirán tratamiento placebo frente a las otras 20 que recibirán distintas dosis de BF.

\section{MATERIAL Y MÉTODOS}

El presente estudio experimental, in vitro, randomizado, intervencionista, fue aprobado por el Comité Ético de Experimentación en Animales por el Hospital Gómez Ulla, Madrid (España).

Durante el experimento, las condiciones de alojamiento animal fueron ofrecidas por el Servicio de Medicina y Cirugía Experimental (SMCEX). Siendo estas confortables, higiénicas, con iluminación natural y artificial, y temperatura controlada entre $20^{\circ} \mathrm{C}$ y $24^{\circ} \mathrm{C}$. La comida y agua empleada durante el ensayo fue ad libitum. La limpieza de las jaulas, se realizaron siguiendo los Procedimientos Normalizados de Trabajo del SMCEX por el personal encargado del animalario y se evitó al máximo cualquier tipo de sufrimiento. Los animales fueron tratados siguiendo lo dispuesto en el Real Decreto 1201/2005, de 10 de octubre, sobre protección de los animales utilizados para experimentación y otros fines científicos.

Los requerimientos mínimos exigidos para la investigación fueron: como criterio de inclusión rata Wistar madura de más 6 semanas y como criterios de exclusión ejemplares en estado de gestación y medicación previa.

Se incluyeron un total de 30 ratas Wistar adultas, de las cuales el $50 \%$ fueron machos y el otro $50 \%$ hembras, con un peso medio de 270 gramos y un rango de [210-330].

Los animales fueron seleccionados de forma aleatoria de las jaulas principales del animalario. Se distribuyeron por sexo y grupo en jaulas de 3 animales como máximo y se etiquetaron adecuadamente.

Para distinguir a cada animal de su jaula, se les pintó en la cola, con un rotulador indeleble marcas identificativas.

Se requirieron tres líneas de animales de experimentación:

- Grupo Control $\left(\mathrm{G}_{\mathrm{O}}\right)$ : sin tratamiento con ácido zoledrónico.

- Grupo $1\left(\mathrm{G}_{1}\right)$ : con tratamiento con Ácido Zoledrónico (ZA) $7,5 \mu \mathrm{g} / \mathrm{Kg}$ subcutáneo, (Zometa ${ }^{\circledR}$, Novartis, Suiza), en los días 0,15 y 30 .

- Grupo $2\left(\mathrm{G}_{2}\right)$ : con tratamiento con Ácido Zoledrónico (ZA) $35 \mu \mathrm{g} / \mathrm{Kg}$ subcutáneo, (Zometa ${ }^{\circledR}$, Novartis, Suiza), en los días 0,15 y 30 .

Se realizó el día 30 la exodoncia del primer molar inferior derecho mediante el siguiente protocolo Figura 1: Primeramente los animales fueron anestesiadas con ketamina (Ketolar ${ }^{\circledR}$ 50, Pfizer, Ireland) y medetomidina (Domtor ${ }^{\circledR}$, Norvet, Finlandia) intramuscular, $75 \mathrm{mg} / \mathrm{Kg}$ y $0,5 \mathrm{mg} / \mathrm{Kg}$ respectivamente. Una vez anestesiados, se colocaron a los animales de forma supina en una tabla de corcho sobre una mesa quirúrgica. A continuación, se inmovilizó la boca, para que quedase abierta, con hilos de sutura fijadas con agujas a la tabla de corcho.

Se colocaron gasas estériles en la zona genital por la posible micción y en la cabeza para una mejor manipulación. Seguidamente se luxó el diente con un Hollenback 3ss (Hu-Friedy®) y se extrajo la pieza mediante unas pinzas mosquito pequeñas rectas (Stainless CEREDA ${ }^{\circledR}$, Germany) realizando pequeños movimientos vestíbulo linguales y rotacionales. A continuación, se introdujo una fresa 
redonda $(\mathrm{D}+\mathrm{Z} \AA \mathrm{CB} 141$ 104.018) de pieza de mano (Kavo® Elecktrotechnisches Werk $\mathrm{GmbH}$ ), $2 \mathrm{~mm}$ desde la cresta alveolar, con el fin de eliminar la mayor cantidad de restos radiculares. Tras la exodoncia se presionó la herida mediante una gasa estéril durante dos minutos

Durante la investigación no se perdió ningún animal, tampoco se suministró antibioterapia. Además, tampoco se cambió la dieta habitual durante el postoperatorio quirúrgico, ya que si se trituraba podía haber riesgo que los animales no lo comiesen, al no estar habituadas a ello.

Los animales fueron sacrificados, siguiendo el Real Decreto $1201 / 2005$, a la cuarta semana post-extracción, mediante métodos que implicaran el mínimo sufrimiento posible con pentobarbital sódico intracardiaco (Dolethal ${ }^{\circledR}$, Vetoquinol S.A. Francia), bajo anestesia previa.

Una vez sacrificado, se procedió a la mandibulectomía correspondiente y obtención de muestras, observándose clínicamente la presencia o ausencia de ONMB según la clasificación de la $\mathrm{AAOMS}^{31}$

Además se procedió a realizar radiografías intraorales laterales (Radiografias Kodak Ultra-speed size 2 DF-58) en todos los grupos, para valorar la aparición esclerosis ósea, reacción perióstica y secuestro óseo. ${ }^{20}$

Seguidamente las muestras fueron introducidas en formaldehído $10 \%$ en recipientes estériles individualizados. Posteriormente fueron sumergidas en un decalcificante (Histofix ${ }^{\circledR}$ decalcifier 3DC, Panreac, Barcelona, España) durante 21 días, y después fueron: cortadas en secciones de $5 \mu$, fijadas con hematosilina-eosina, y observadas mediante un microscopio óptico (Eclipse 50i; Nikon YTHR,Tokyo,Japan) para valorar la aparición de necrosis e inflamación ${ }^{21}$
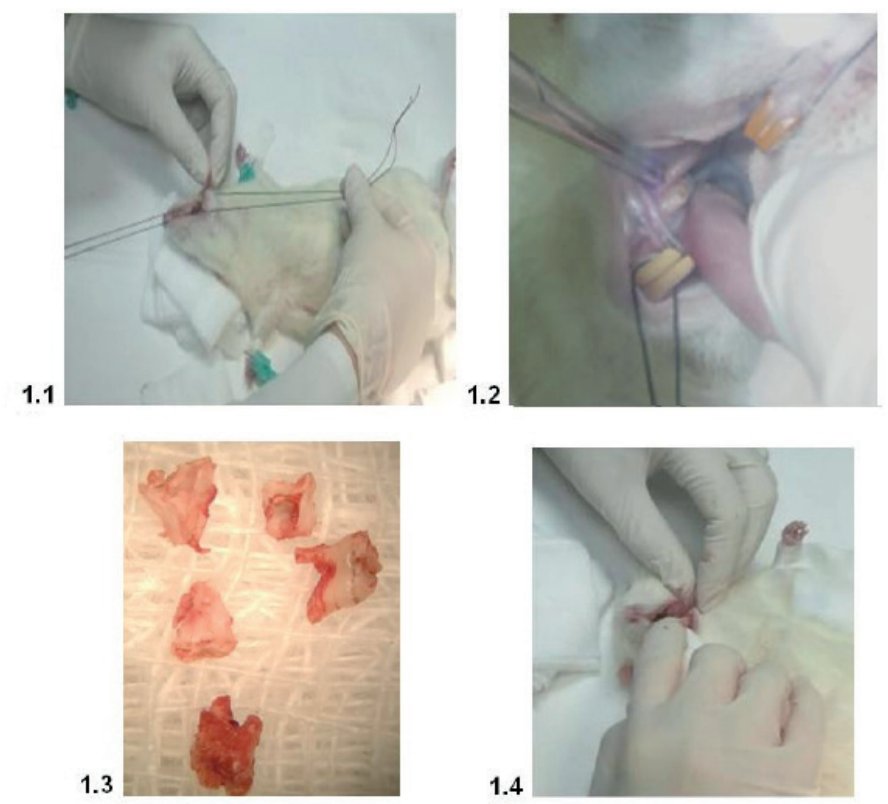

Figura 1. Procedimiento quirúrgico. 1.1. Inmovilización de la boca con hilos de sutura fijadas con agujas a la tabla de corcho.1.2. Extracción del molar inferior con pinzas mosquito y perforación del alveolo $2 \mathrm{~mm}$.1.3. Molares inferiores extraídos. 1.4. Presión sobre la herida mediante una gasa estéril durante un par de minutos.
Las imágenes digitales del microscopio óptico (magnificación $2 \mathrm{x} / 0.06$ ), se obtuvieron a través del sistema "Digital Sight DS-L1" (Nikon, Japan) y de las radiografías intraorales con el sistema " $\mathrm{Cy}-$ ber-Shot" (Sony).

Los datos estadísticos se realizaron a partir del programa, IBM Corp. Released 2013. IBM SPSS Statistic for Windows, versión 22.0 Armonk, NY: IBM Corp, donde se utilizó para las variables cualitativas el test estadístico de chi-cuadrado $\mathrm{X}^{2}\left(\mathrm{H}_{1}, \mathrm{H}_{2}\right)$. El valor de " $p$ " inferior a 0,05 fue considerado estadísticamente significativo para todos los test.

Se analizaron las siguientes variables clínicas, histopatológicas y radiológicas para realizar los test estadísticos con chi-cuadrado $\mathrm{X}^{2}$ :

- Clínico: Estadios según la AAOMS ${ }^{31}$ :

- 0 : Sin evidencias de hueso expuesto en pacientes con tratamiento de BF orales o intravenosos.

- 1: Presencia de hueso expuesto normal o necrótico en pacientes asintomáticos sin evidencia de infección o de inflamación significativa del tejido blando de los maxilares.

- 2: Presencia de hueso expuesto normal o necrótico en pacientes con dolor, con signos evidentes de infección secundaria e inflamación del tejido blando subyacente.

- 3: Presencia de hueso expuesto normal o necrótico en pacientes con dolor, inflamación, infección y uno o más de los siguientes signos: fractura patológica, fístula extraoral u osteolisis que se extiende al borde inferior.

- Histopatológico:

- Necrosis: 0 si no hay presencia. 1 cuando hay presencia de hueso necrótico con o sin secuestro óseo y un marcado infiltrado inflamatorio mixto ${ }^{21}$.

- Inflamación: 0 no, 1 si con infiltrado inflamatorio ${ }^{21}$.

- Radiográfico: 0 no hay necrosis, 1 si hay necrosis (esclerosis ósea, reacción perióstica y secuestro óseo ${ }^{20}$ ).

\section{RESULTADOS}

La distribución de la muestra según los fármacos, el 33,3\% eran placebo $\left(\mathrm{G}_{0}\right)$, el $33,3 \% 7,5 \mu \mathrm{g} / \mathrm{Kg}$ de ácido zoledrónico y el otro $33,3 \% 35 \mu \mathrm{g} / \mathrm{Kg}$ de ácido zoledrónico.

Clínicamente se observó que tras realizar una exodoncia y esperar un período de 30 días para la cicatrización, 8 animales $[26,6 \%$ del estudio; 3 animales en el grupo $\mathrm{G}_{1}(10 \%$ del estudio) y 5 animales en el grupo $\mathrm{G}_{2}(16,6 \%)$ ] presentaban un hueso expuesto sin evidencia de infección ni de inflamación del tejido blando de los maxilares compatible con un estadio I de ONMB en humanos. Los resultados presentaron significación estadística $(\mathrm{p}<0,011)$ Tabla 2 .

Histopatológicamente, no se pudo evidenciar hueso necrótico ni la presencia de infiltrado inflamatorio, $(\mathrm{p}<0,001)$ Tabla 2 y Figura 2.

Radiográficamente, las mandíbulas no mostraron signos radiológicos de esclerosis ósea, reacción perióstica y secuestro óseo Tabla 2, Figura 3. Diagnosticándose en todos los grupos " 0 ", siendo $\mathrm{p}<0,001$.

\section{DISCUSIÓN}

La rata, después del ratón, es el animal de experimentación más utilizado en investigaciones biomédicas, especialmente en fisiolo- 
Tabla 2. Resultado de las variables del estudio.

\begin{tabular}{|ccccc|}
\hline & Clínica ONMB & Histopatológica Necrosis & $\begin{array}{c}\text { Histopatológica } \\
\text { Inflamación }\end{array}$ & Radiológica ONMB \\
\hline Grupo $\mathrm{G}_{\mathrm{O}}$ & $\mathrm{n}=0$ & $\mathrm{n}=0$ & $\mathrm{n}=0$ & $\mathrm{n}=0$ \\
Grupo $\mathrm{G}_{1}$ & $\mathrm{n}=3(10 \%)$ & $\mathrm{n}=0$ & $\mathrm{n}=0$ & $\mathrm{n}=0$ \\
Grupo $\mathrm{G}_{2}$ & $\mathrm{n}=5(16,6 \%)$ & $\mathrm{n}=0$ & $\mathrm{n}=0$ & $\mathrm{n}=0$ \\
\hline Valor $\mathrm{p}$ & $<0,011$ & $<0,001$ & $<0,001$ & $<0,001$ \\
\hline
\end{tabular}

n: número de animales

$\mathrm{G}_{0}$ : Control, sin tratamiento con Zometa ${ }^{\circledR}$ (Novartis, Suiza).

$\mathrm{G}_{1}$ :Tratamiento con $7,5 \mu \mathrm{g} / \mathrm{Kg}$ subcutáneo, en los días 0,15 y 30 .

$\mathrm{G}_{2}$ :Tratamiento con Zometa $35 \mu \mathrm{g} / \mathrm{Kg}$ subcutáneo, en los días 0,15 y 30 .

gía, toxicología, farmacología, comportamiento, inmunología y oncología $^{33-34}$. En este estudio se utilizó la rata y no el ratón por su mayor tamaño, mejor manipulación y visualización dentaria durante la extracción dental.

Con el fin de entender mejor la fisiopatología de la ONMB y poder desarrollar un enfoque terapéutico. Se realizó este estudio en ratas Wistar, por ser esta un modelo de animal fiable, reproducible y de fácil mantenimiento ${ }^{33-34}$.

Según Gottardello et cols ${ }^{35}$ y Vignery et cols $^{36}$, la exodoncia de molares en ratas es más apropiada que en otros animales, debido a la ausencia de cartílago y el corto periodo de curación.

En este estudio, al igual que en los de otros autores ${ }^{37-41}$, se extrajeron los molares inferiores, por ser el procedimiento quirúrgico más habitual y fácil de normalizar. Los molares superiores en la rata Wistar están en contacto con el seno maxilar y los incisivos son imprescindibles para la alimentación y calidad de vida. También, se eligió el molar inferior, porque la ONMB en humanos, aparece más frecuentemente en la mandíbula que en el maxilar ${ }^{11,12,22,25}$.

Al igual que otros estudios, se eligieron ratas adultas y no jóvenes, porque en humanos el riesgo de aparición de ONMB es más frecuente en personas adultas que en jóvenes ${ }^{22}$. La mandíbula de estos animales tiene gran densidad ósea y resistencia, y las raíces tienden a presentar cementosis perirradicular cuando son adultas. Esto hace que la exodoncia de los molares sea difícil, con propensión a la fractura radicular ${ }^{35,42,43}$.

En la bibliografía actual los autores suministran el ZA, en estos animales, subcutáneamente $\mathrm{e}^{37,39,41,44}$, o por vía intravenosa ${ }^{40,42,45-47}$. Aunque no haya estandarizada una dosis concreta de ZA para su uso

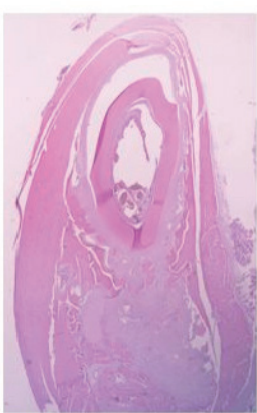

2.1

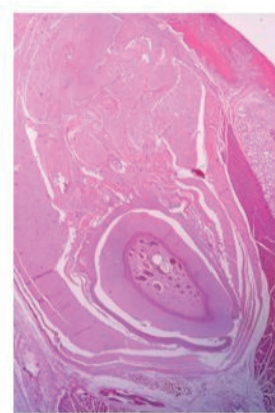

2.2

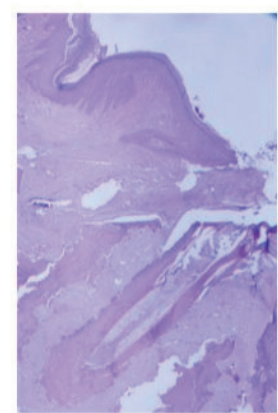

2.3
Figura 2. Estudio histopatológico.2.1 Corte histopatológico, con magnificación 0’06, en una rata del estudio experimental G0.2.2 Corte histopatológico, con magnificación 0'06, en una rata del estudio experimental G1.2.3 Corte histopatológico, con magnificación 0'06, en una rata del estudio experimental $G 2$. en ratas en la literatura ${ }^{39,44}$, se administró la dosis empleada Mehmet et cols ${ }^{37}$ y Hokugo et cols ${ }^{45}$ en ratas Wistar.

Según la revisión bibliográfica estudiada, existen diversos métodos para realizar la extracción dentaria en ratas (Tabla 3). En el presente estudio, para realizar la exodoncia se utilizó la técnica de Pietrokovski et cols. ${ }^{43}$ y Johansen et cols. ${ }^{42}$ : luxando la pieza y posteriormente extrayéndola con pinzas mosquito. Sin embargo se observó que la mayoría de las raíces se fracturaban al igual que Mehmet et $\operatorname{cols}^{37} \mathrm{y}$ Sonis et $\operatorname{cols}^{39} \mathrm{y}$ por ello se procedió a realizar la técnica de Marino et cols $s^{47}$, realizando una perforación de dos milímetros del alveolo desde la cresta alveolar, con el fin de eliminar mayor cantidad de restos radiculares.

Según Pietrokovsky et cols ${ }^{43}$, los restos radiculares interfieren en el proceso de reparación óptima del alveolo, mientras Johansen et cols $s^{42}$, opinaban que podían dar información interesante acerca de las reacciones en el periodonto de la raíz, y por ello no las eliminó, para evitar el riesgo quirúrgico adicional que pudiera influir en la reparación ósea.

Tras realizar la exodoncia dentaria, hay autores que prefieren suturar la herida para evitar la hemorragia ${ }^{35}$, sin embargo en nuestro estudio al igual que Biasotto et cols ${ }^{46}$, se presionó la herida con una gasa durante un par de minutos.

En general los animales mostraron buena hemostasia y rápida recuperación de la anestesia ${ }^{37}$, sin embargo puede ocurrir hemorra-
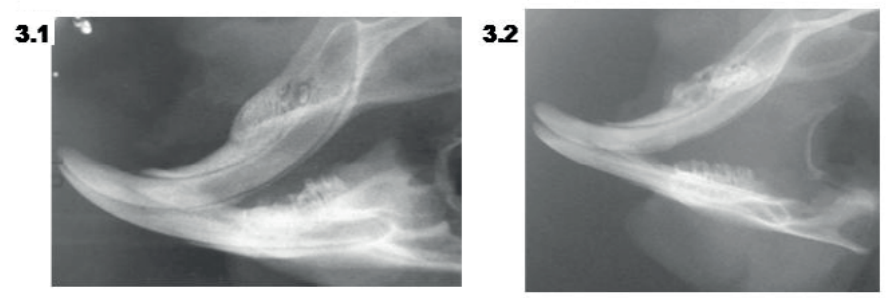

3.3

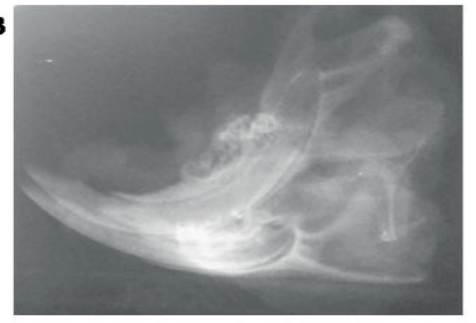

Figura 3. Estudio radiográfico.3.1. Radiografía lateral postmortem de la mandibula de una rata Wistar sana en G0.3.2. Radiografía lateral postmortem de la mandíbula de una rata Wistar sana en G1.3.3 Radiografía lateral postmortem de la mandibula de una rata Wistar en $G 2$. 
Tabla 3. Procedimientos quirúrgicos para la extracción dentaria en ratas.

\begin{tabular}{|ll|}
\hline \multicolumn{1}{|c|}{ Autor/es } & \\
\hline $\begin{array}{l}\text { Mehmet et cols }{ }^{37} \text {, Abtahi } \\
\text { et cols }{ }^{50}, \text { Hokugo et cols }{ }^{47} \\
\text { Johansen } \text { et cols }{ }^{42}, \\
\text { Pietrokovski } \text { et cols }{ }^{43}\end{array}$ & Mediante un explorador dental afilado se luxa la pieza y actúa como fórceps. \\
Gottardello et cols ${ }^{35}$ & Luxación mediante cincel o escalador y posterior extracción con pinzas mosquito. \\
& $\begin{array}{l}\text { Luxación mediante hollenback, odontosección posterior de la pieza mediante una fresa de carburo y } \\
\text { extracción de las raíces con pinzas mosquito }\end{array}$ \\
Marino et cols ${ }^{47}$ & $\begin{array}{l}\text { Luxación mediante un excavador, odontosección posterior de la pieza mediante una fresa de carburo, } \\
\text { extracción de las raíces con pinzas mosquito y si quedara algún resto radicular introducir 2mm la fresa desde } \\
\text { la unión amelo-cementaria. }\end{array}$ \\
Biasotto et cols ${ }^{46}$ & $\begin{array}{l}\text { Perforación del diente mediante una fresa de acero inoxidable, eliminar pieza con elevador dental delgado y } \\
\text { continuar perforando hasta alcanzar } 4 \text { mm de diámetro de defecto óseo. }\end{array}$ \\
\hline
\end{tabular}

Tabla 4. Proceso de cicatrización del alveolo en ratas tras realizar una exodoncia dentaria ${ }^{42-43}$.

\begin{tabular}{|c|c|c|}
\hline Día & Proceso & Observaciones \\
\hline $1^{\mathrm{o}}$ & Formación de coágulo & $\begin{array}{l}\text { El alveolo contiene células sanguíneas, fibrina, restos de fibras periodontales y células del } \\
\text { tejido conjuntivo a lo largo de la pared alveolar. }\end{array}$ \\
\hline $2^{\circ}$ & $\begin{array}{l}\text { Reemplazo del coágulo } \\
\text { por tejido de granulación }\end{array}$ & $\begin{array}{l}\text { Se produce la formación de tejido de granulación y comienzo de hueso neoformado (signos } \\
\text { de aposición y reabsorción ósea). }\end{array}$ \\
\hline $3^{\circ}$ & $\begin{array}{l}\text { Sustitución del tejido de granulación por tejido } \\
\text { conectivo y epitelización }\end{array}$ & $\begin{array}{l}\text { El alveolo esta rellenado de tejido de granulación rellenado, macrófagos, fibroblastos y } \\
\text { signos de aposición y reabsorción ósea. La superficie del epitelio casi cubría la herida. }\end{array}$ \\
\hline $4^{\circ}-5^{\circ}$ & $\begin{array}{l}\text { Aparición de tejido osteoide } \\
\text { en la base del alvéolo }\end{array}$ & Intensa formación de nuevo hueso y surgen células osteoprogenitoras. \\
\hline $6^{\circ}$ & $\begin{array}{l}\text { Relleno de los dos tercios apicales de hueso } \\
\text { trabecular }\end{array}$ & $\begin{array}{l}\text { El alvéolo se encuentra rellenado de hueso nuevo. El epitelio cubre completamente y } \\
\text { mayor cantidad de células osteoprogenitoras (inicio en la cresta). }\end{array}$ \\
\hline $11^{\mathrm{o}}$ & Epitelio queratinizado & Formación de hueso trabeculado y epitelio está totalmente queratinizado. \\
\hline
\end{tabular}

gia profusa como consecuencia de la extracción dental del tercer molar, porque los vasos del canal dentario son de gran tamaño ${ }^{35}$.

En la cicatrización de los alvéolos en ratas sanas tras una exodoncia, el neo hueso rellena la cavidad y el epitelio muestra total queratinización en la $2^{\circ}$ semana postextracción ${ }^{42-43}$ (Tabla 4). Se decidió, al igual que en otros estudios con ZA, sacrificar a los animales a las 4 semanas postextracción ${ }^{36-38}$. Se esperaron estas 4 semanas, dos veces el tiempo de lo necesario, para tener certeza que la cicatrización se ha completado. Se consideró anormal y signo de ONMB la presencia de alteraciones en la cicatrización.

Distintos estudios con ratas Wistar con tratamiento de ZA, contemplaron clínicamente retraso en la cicatrización y ulceraciones, inflamación y necrosis ${ }^{37,40-41,46}$, como igualmente ocurre en huma$\operatorname{nos}^{7,11-13,15}$. Los bifosfonatos liberados son tóxicos para el epitelio y pueden inhibir en la proliferación y la cicatrización ${ }^{48}$. En nuestro estudio la epitelización del grupo $\mathrm{G}_{\mathrm{O}}$ fue completa a los 30 días y sin diferencias con el tejido contiguo. En los grupos con tratamiento $\mathrm{G}_{1}-\mathrm{G}_{2}$, se observó que la epitelización no fue completa y el hueso alveolar estaba expuesto en un número con significación estadística. La frecuencia de aparición manifestó ser dosis dependiente. Los resultados mostraron significación estadística $p<0,011$. Se muestran en la tabla 2. La presencia de estas anomalías es compatible con el estadio I de la $\mathrm{AAOMS}^{31}$.

No se observaron signos de inflamación y necrosis en los estudios radiográficos e histológicos, siendo el resultado $p<0,001$ en todos los grupos. Se muestran en la tabla 2. Estos resultados difieren de los de Mehmet et cols. ${ }^{37}$, en ellos sí se observó histopatológicamente signos de ONMB suministrando $7,5 \mu \mathrm{g} / \mathrm{Kg}$ (necrosis $\mathrm{p}>0,015$ e inflamación p>0,0001).
Agaçayak et cols.$^{41}$ observó en su estudio ONMB radiológica. Sin embargo, en nuestro estudio no se observaron radiográficamente zonas con patrón moteado, sin compromiso de las corticales, eriostio o lámina dura. En las mandíbulas diagnosticadas clínicamente en estadio I de la AAOMS, no se observaron otros signos de ONMB. Tampoco se observaron alteraciones óseas características de estadios más avanzados de osteonecrosis. Nuevos estudios se hacen necesarios para comprender los fenómenos encontrados.

Los animales, al igual que otros estudios, perdieron peso tras la exodoncia ${ }^{42,49,50}$, sin embargo en nuestro trabajo, desde la extracción hasta el sacrificio lo ganaron ${ }^{42,43,50}$.

\section{CONCLUSIONES}

En nuestro modelo de investigación animal (rata wistar) se observó clínicamente que la suministración de ácido zoledrónico subcutáneamente durante cuatro semanas y 30 días después de la exodoncia del primer molar inferior derecho, aparece hueso expuesto sin la epitelización completa. Situación compatible con un estadio I de osteonecrosis oral en humanos según la American Association of Oral Maxillofacial Súrgeons. Estos signos clínicos mostraron ser dosis dependiente.

Con las dosis y tiempos empleados en nuestro estudio no hubo signos radiológicos, ni anatomopatologicos de ONMB.

Se necesitan más estudios con bifosfonatos, en diferentes dosis, vías de administración e intervalos, para comprender mejor la aparición de ONMB. 


\section{BIBLIOGRAFÍA}

1. Rogers MJ, Gordon S, Benford HL, Coxon FP, Luckman SP, Monkkonen J et al. Cellular and molecular mechanisms of action of bisphosphonates. Cancer 2000;86: 2961-78.

2. Wood J, Bonjean K, Ruetz S, Bellahcéne A, Devy L, Foidart JM et al. Novel antiangiogenic effects of the bisphosphonate compound zoledronic acid. J Pharmacol ExpTher 2002; 302:1055-61

3. Fournier P, Boissier S, Filleur S, Guglielmi J, Cabon F, Colombel M, et al. Bisphosphonates inhibit angiogenesis in vitro and testosterone-stimulated vascular regrowth in the ventral prostate in castrated rats. Cancer Res. 2002;62: 6538-44.

4. Liccata AA. Discovery, clinical development, and therapeutic uses of biphosphonates. Ann Pharmacother. 2005;39 (4):668-77.

5. Coleman RE. Future directions in the treatment and prevention of bone metastases. Am J Clin Oncol.2002;25: 32-8.

6. Melo MD, Obeid G. Osteonecrosis of the jaws in patients with a history of receiving bisphosphonate therapy: strategies for prevention and early recognition. $\mathrm{J}$ Am Dent Assoc. 2005 Dec;136(12):1675-81.

7. Drake MT, Clarke BL, Khosla S. Bisphosphonates: mechanism of action and role in clinical practice. Mayo Clin Proc. 2008;83(9):1032-45.

8. Woo SB, Hellstein JW, Kalmar JR. Systematic Review: Biphosphonates and Osteonecrosis of the Jaws. Ann Intern Med.2006; 144:753-761.

9. Fleisch H. Bisphosphonates: Mechanisms of Action. Endocrine Reviews.1998;19(1):80-100.

10. Arrabal Martín M, Valle Díaz de la Guardia F, Jiménez Pacheco A, López León V, Arrabal Polo MA, Zuluaga Gómez A. Tratamiento de la litiasis renal con bifosfonatos. Arch Esp Urol. 2007;60(7):745-54

11. Marx RE. Pamindronate (Aredia) and zoledronate (Zometa) induced avascular necrosis of the jaws: a growing epidemic. J Oral Maxillofac Surg. 2003;61:1115-17.

12. Ruggiero SL, Mehrotra B, Rosenberg TJ, Engroff SL. Osteonecrosis of the jaws associated with the use of bisphosphonates:a review of 63 cases. J Oral Maxillofac Surg.2004;62:527-34.

13. Senel FC, Kadioglu Duman M, Muci E, Cankaya M, Pampu AA, Ersoz S, Gunhan $\mathrm{O}$. Jaw bone changes in rats after treatment with zoledronate and pamidronate. Oral Surg Oral Med Oral Pathol Oral Radiol Endod. 2010;109(3):385-91.

14. Roelofs AJ, Thompson K, Gordon S, Rogers MJ. Molecular mechanisms of action of bisphosphonates: current status. Clin Cancer Res. 2006;12:20.

15. Pazianas M, Miller P, Blumentals WA.A review of the literature on osteonecrosis of the jaw in patients with osteoporosis treated with oral bisphosphonates: prevalence,risk factors, and clinical characteristics. Clin Ther.2007;29:1548-58.

16. Jeremy Allgrove. Biphosphonates. Archives of Disease in Childhood. 1997;76:73-75.

17. Migliorati CA, Casiglia J. Managing the care of patients with bisphosphonateassociated osteonecrosis. J Am Dent Assoc.2005;136(12):1658-67.

18. Migliorati CA. Biphosphonates and oral cavitynavascular necrosis of bone. J Clin Oncol 2003;21(22):4253-4.

19. Ruggiero SL, Dodson TB, Assael LA, Landesberg R, Marx RE, Mehrotra B; Task Force on Bisphosphonate-Related Osteonecrosis of the Jaws, American Association of Oral and Maxillofacial Surgeons. American Association of Oral and Maxillofacial Surgeons position paper on bisphosphonate-related osteonecrosis of the jaw - 2009 update. Aust Endod J. 2009; 35(3):119-30.

20. Estilo CL, Van Poznak CH, Wiliams T, Bohle GC, Lwin PT, Zhou Q, et al. Osteonecrosis of the maxilla and mandible in patients with advanced cancer treated with bisphosphonate therapy. Oncologist 2008; 13(8):911- 20.

21. Favia G, Pilolli GP, Maiorano E. Histologic and histomorphometric features of bisphosphonate-related osteonecrosis of the jaws: an analysis of 31 cases with confocal laser scanning microscopy. Bone. 2009; 45: 406-13.

22. Escobar López EA, López López J, Marques Soares MS, Chimenos Küstner E. Osteonecrosis de los maxilares asociada a bifosfonatos: revisión sistemática. Av. Odontoestomatol 2007;23(2):91-101

23. Dixon RB, Tricker ND, Garetto LP. Bone turnover in elderly canine mandible and tibia.J Dent Res 1997; 76:336.

24. Marx RE, Sawatari Y, Fortin M, Broumand V. Biphosphonate-induced exposed bone (osteonecrosis/osteopetrosis) of the jaws: risk factors, recognition, prevention, and treatment. J Oral Maxillofac Surg 2005; 63:1567-751.

25. Badros A, Weikel D, Salama A, Goloubeva O, Schneider A, Rapoport A, Fenton R, Gahres N, Sausville E, Ord R, Meiller T. Osteonecrosis of the jaw in multiple myeloma patients: clinical features and risk factors. J Clin Oncol.2006;24(6):945-52.
26. Jiménez Soriano Y, Bagan JV. Los bifosfonatos, nueva causa de osteonecrosis maxilar por fármacos: situación actual. Med Oral Patol Oral Cir Bucal 2005; 10 (2): 88-91.

27. Hoff AO, Toth BB, Altundag K, Guarneri V, Adamus A, Nooka AK et al. Osteonecrosis of the jaw in patients receiving intravenous bisphosphonate therapy $\mathrm{J}$ Clin Oncol. 2006: 24; 8528.

28. Reid, I. R., Cornish, J. Nat. Rev. Rheumatol. 2012:8;90-6.

29. Expert Panel Recommendations for the Prevention, Diagnosis, and Treatment of Osteonecrosis of the Jaws: June 2004. Professional Education Material. East Hanover, NJ: Novartis; 2004.

30. Migliorati CA, Casiglia J, Epstein J, Jacobsen PL,Siegel MA, Woo SB. Managing the care of patients with bisphosphonate-associated osteonecrosis.Journal American Dental Association 2005; 136:1658-68.

31. American Association of Oral and Maxillofacial Surgeons position paper on bisphosphonate-related osteonecrosis of the jaws. J Oral Maxillofac Surg 2007;65: 369-76.

32. Aragonès Forès R, Orozco López P. Abordaje de la osteoporosis en la atención primaria en España (estudio ABOPAP-2000). Aten Primaria 2002; 30:350-6.

33. Atchley W, Fitch W. Genes trees and the origins of inbread strain of mice. Science 1991; 254: 554-8.

34. Clause BT. The Wistar Intitute Archives: Rats (Not Mice) and History. Mendel Newsl.1998; 7:2-7.

35. Gottardello KZ, Rogério da Silva J, Jacks J. A new method for extraction of mandibular first molars in rats- Braz J Oral Sci 2007:6(21);1344-8.

36. Vignery A, Baron R. Dynamic histomorphometry of alveolar bone remodeling in the adult rat. Anat Rec. 1980; 96: 191-200.

37. Mehmet A.E, Abdulkadir B.C,Sabri C.I, Sabit D, Merva S, Cetin K et al. Extraction socket healing in rats treated with bisphosphonate: Animal model for bisphosphonate related osteonecrosis of jaws in multiple myeloma patientes. Med oral patol Oral Cir Bucal 2011; 16(7);879-83.

38. Marino KL, Zakhary I, Abdelsayed RA, Carter JA, O'Neill JC, Khashaba RM et al. Development of a rat model of bisphosphonate-related osteonecrosis of the jaw (BRONJ). J Oral Implantol. 2012; 38:511-8.

39. Sonis ST, Watkins BA, Lyng GD, Lerman MA, Anderson KC. Bony changes in the jaws of rats treated with zoledronic acid and dexamethasone before dental extractions mimic bisphosphonate-related osteonecrosis in cancer patients. Oral Oncol. 2009; 45:164-72.

40. Sarkarat F, Motamedi MHK, Jahanbani J, Sepehri D, Kahali R, Nematollahi Z. Platelet-Rich Plasma in Treatment of Zoledronic Acid-Induced Bisphosphonaterelated Osteonecrosis of the Jaws. Trauma Mon. 20141; 19(2): 17196.

41. Agaçayak KS, Yuksel H, Atilgan S, Koparal M, Uçan MC, Özgöz M, et al. Experimental investigation of relationship between trauma and bisphosphonate-related osteonecrosis. Níger J Clin Pract.2014; 17 (5): 559-64.

42. Johansen JR. Repair of the post-extaccion alveolus in the Wistar rat. A histologic and autoradiographic study. Acta Odontolol Scand. 1970; 28:441-6179.

43. Pietrokovski J, Massler M. Ridge remodeling after tooth extraction in rats. J Dent Res. 1967:46;222-31.

44. Kuroshima S, Mecano RB,Tanoue R, Koi K, Yamashita J. Distinctive tooth- extraction socket healing: bisphosphonate versus parathyroid hormone therapy .J Periodontol 2014; 85(1):24-33.

45. Hokugo A, Christensen R, Chung EM, Sung EC, Felsenfeld AL, Sayre JW et al. Increased Prevalence of Bisphosphonate-Related Osteonecrosis of the Jaw with Vitamin D Deficiency in Rats. Journal of Bone and Mineral Research 2010; 25(6):1337-49.

46. Biasotto M, Chiandussi S, Zacchigna S, Moimas S, Dore F, Pozzato G et al. A novel animal model to study non-spontaneous bisphosphonates osteonecrosis of jaw. J Oral Pathol Med 2010; 39: 390-6.

47. Marino KL, Zakhary I, Abdelsayed RA, Carter JA, O’Neill JC, Khashaba RM, Elsalanty M, Stevens MR, Borke JL. Development of a rat model of bisphosphonate-related osteonecrosis of the jaw (BRONJ). J Oral Implantol. 2012; 38:511-8.

48. Reid IR, Bolland MJ, Grey AB. Is bisphosphonate-associated osteonecrosis of the jaw caused by soft tissue toxicity? Bone. 2007; 41:318-20.

49. Conte Neto N, Spolidorio LC, Andrade CR, Bastos AS, Guimaraes M, Marcantonio E Jr. Experimental development of biphosphonate-related osteonecrosis of the jaws in rodents. Int J Exp Path. 2013; 94:65-73.

50. Abtahi J, Agholme F, Sandberg O, Aspenberg P. Bisphosphonate-induced osteonecrosis of the jaw in a rat model arises first after the bone has become exposed: no primary necrosis in unexposed bone. J Oral Pathol Med 2012;41:494-99. 